\title{
The effectiveness and cost-effectiveness of treatments for idiopathic pulmonary fibrosis: systematic review, network meta-analysis and health economic evaluation
}

Emma Loveman ${ }^{1 *}$, Vicky R Copley ${ }^{1}$, Jill L Colquitt ${ }^{1}$, David A Scott ${ }^{2}$, Andy J Clegg ${ }^{1}$, Jeremy Jones ${ }^{1}$, Katherine MA O'Reilly ${ }^{3}$, Sally Singh ${ }^{4}$, Claudia Bausewein ${ }^{5}$ and Athol Wells ${ }^{6}$

\begin{abstract}
Background: Idiopathic pulmonary fibrosis (IPF) is a life-limiting lung disease with considerable impact on patients and carers as the disease progresses. Currently few treatments are available. We aimed to evaluate the clinical and cost-effectiveness of available treatments for IPF.

Methods: Systematic reviews of clinical effectiveness, quality of life and cost effectiveness were undertaken. Eleven bibliographic databases were searched from inception to July 2013 and studies were assessed for eligibility against a set of pre-defined criteria. Two reviewers screened references, extracted data from included studies and appraised their quality. An advisory group was consulted about the choice of interventions. A narrative review was undertaken and where feasible fixed effect and random effects meta-analysis were undertaken including a network

meta-analysis (NMA).

A decision-analytic Markov model was developed to estimate cost-effectiveness of pharmacological treatments for IPF. Following best practice recommendations, the model perspective was of the national health service and personal social services, a discount rate of 3.5\% for costs and health benefits was applied and outcomes were expressed as cost per quality adjusted life-year gained. Parameter values were obtained from the NMA and systematic reviews. Sensitivity analyses were undertaken.
\end{abstract}

Results: Fourteen studies were included in the review of clinical effectiveness, of which one evaluated azathioprine, three $\mathrm{N}$-acetylcysteine [NAC] (alone or in combination), four pirfenidone, one nintedanib, one sildenafil, one thalidomide, two pulmonary rehabilitation, and one a disease management programme. Study quality was generally good. Evidence suggests that some effective treatments are available. In NMA only nintedanib and pirfenidone show statistically significant improvements. The model results show increased survival for five pharmacological treatments (NAC triple therapy, inhaled NAC, nintedanib, pirfenidone, and sildenafil) compared with best supportive care, at increased cost. Only inhaled NAC was cost-effective at current willingness to pay thresholds but it may not be clinically effective.

Conclusions: Few interventions have any statistically significant effect and the cost-effectiveness of treatments is uncertain. A lack of studies on palliative care approaches was identified and there is a need for further research into pulmonary rehabilitation and thalidomide in particular. A well conducted RCT on inhaled NAC therapy should also be considered.

Keywords: Idiopathic pulmonary fibrosis, Systematic review, Meta-analysis, Cost-effectiveness

\footnotetext{
* Correspondence: emma.loveman@soton.ac.uk

'Southampton Health Technology Assessments Centre (SHTAC), University of Southampton, Southampton, UK

Full list of author information is available at the end of the article
} 


\section{Background}

Idiopathic pulmonary fibrosis (IPF) is a debilitating respiratory condition for which there is no cure. IPF is characterised by aberrant wound healing in which excessive (and perhaps abnormal) extracellular matrix is deposited in the lung thereby distorting the architecture and disrupting function. This lung injury and scarring eventually leads to a decline in lung function which culminates in respiratory failure [1]. Shortness of breath on exercise and a chronic dry cough are the prominent symptoms [2]. IPF is known to affect males more than females and in particular affects people over 60 years of age. The prevalence of IPF is increasing, although the cause of this increase is uncertain, [3,4] and with a poor prognosis (estimated mean survival of between $2-5$ years) IPF has become an area of focus with recent UK national guidelines on the diagnosis and management of IPF published, [5] and international consensus guidelines [6] due to be updated in 2014.

IPF is a difficult condition to manage, particularly in the latter stages. Early and accurate diagnosis is important to maximise the potential for a better outcome but there is an unmet need with few recommended treatments [5]. In the UK all patients should be given best supportive care (BSC) from the point of diagnosis, which includes information and support, symptom relief, management of co-morbidities, withdrawal of ineffective therapies and end-of-life care. In addition individuals should be assessed for pulmonary rehabilitation if appropriate and have a clinical nurse specialist available to them [5]. For those without contraindications lung transplantation should be considered as this is the only treatment shown to improve survival [7]. However, with donor organs being in short supply there is a need for alternative treatments that aim to modify the disease and prolong survival. Few treatments are available to the clinician and patient currently, and the evidence for the effectiveness of such treatments is unclear. We aimed to evaluate the current state of the clinical and cost-effectiveness of treatments for people with IPF through three systematic reviews, a network meta-analysis (NMA) and economic modelling.

\section{Methods}

The a priori methods for this evidence synthesis are described in the research protocol which is registered with PROSPERO (reference: 42012002116). Search strategies were developed and applied to 11 electronic bibliographic databases (including the Cochrane library, MEDLINE and EMBASE) from inception to July 2013 with no language restrictions. Bibliographies of retrieved papers were screened and experts contacted to identify additional studies. Systematic reviews were undertaken of clinical effectiveness (including only randomised controlled trials [RCTs] and controlled clinical trials [CCTs]), economic evaluations and health related quality of life (HRQoL) studies. Eligible participants were those with a diagnosis of IPF and includable interventions were as deemed relevant by a clinical and patient advisory group. Best supportive care, placebo or any of the interventions were eligible as comparators and outcomes of relevance included measures of survival, measures of symptoms (breathlessness, cough), HRQoL, lung function, exercise performance, adverse events and measures of costs and cost-effectiveness. Studies reporting HRQoL in people with IPF were eligible for inclusion if they used either generic preference-based measures or the St Georges Respiratory Questionnaire (SGRQ) which is a disease specific instrument used in IPF. Other disease specific instruments were not eligible for inclusion as there are currently no methods to map results of these to utility measures required for economic evaluation.

Titles and abstracts were screened for potential eligibility by two reviewers using a pre-defined inclusion criteria, retrieved articles were assessed for eligibility, data were extracted and methodological quality assessed by one reviewer and checked by a second. Study quality was assessed using recognised methods [8-10]. For the review of clinical effectiveness we developed a check-list to assess the methodological quality of the studies based on the criteria recommended by the Centre for Reviews and Dissemination, [8] (Quality assessment/risk of bias section) and summarised the risk of bias (as per Cochrane collaboration recommendations [11]) within each study according to the risk of selection bias. We developed a check-list to assess the methodological quality of the cost effectiveness studies based on the check-list of Drummond and colleagues [9] and recommendations by Phillips and colleagues [10]. Data items extracted included study details (design, follow-up, funding), participant details (numbers, eligibility, characteristics), intervention details (including dose and duration of treatment), outcomes reported and results. Narrative syntheses were undertaken and in the review of clinical effectiveness meta-analysis was performed where appropriate with heterogeneity assessed. FVC was measured on two continuous scales and these were meta-analysed using the standardised mean difference (SMD). A NMA focusing on pharmacological treatments for IPF and assessing forced vital capacity (FVC) endpoints was undertaken [12].The NMA focused on FVC as it is correlated with disease progression [6] and was therefore relevant to the economic model. For FVC endpoints the NMA used the SMD in a Bayesian framework using code adapted from published sources [13]. Vague normal priors were used for the treatment effects and a vague uniform prior for the random effect standard deviation. Model code is provided in the Additional file 1. Fixed and random effects models were applied with best model fit determined using the deviance information criterion (DIC). The SMDs output from the NMA were then 
converted to $\log$ odds ratios using standard methods for ease of interpretation within the context of an NMA [14].

\section{Quality assessment/risk of bias}

1. Was the method used to generate random allocations adequate?

2. Was the allocation adequately concealed?

3. Were the groups similar at the outset of the study in terms of prognostic factors

4. Was the care provider blinded?

5. Was the patient blinded?

6. Were outcome assessors blinded to the treatment allocation?

7. i) Were there any unexpected imbalances in drop-outs between groups? ii) If so, were they explained or adjusted for?

8. Is there any evidence to suggest that the authors measured more outcomes than they reported?

9. i) Did the analysis include an intention to treat analysis? ii) If so, was this defined?

10. i) Did the analysis account for missing data? ii) If so, were the methods appropriate?

A decision-analytic model was developed to compare the cost-effectiveness of pharmacological interventions in patients with initially unprogressed IPF. The model perspective is that of the UK National Health Service and Personal Social Services. The model structure was informed by the available literature and expert opinion on the clinical progression of the disease. It uses four distinct health states: unprogressed IPF; progressed IPF; lung-transplant; and dead. Health states except death are associated with a HRQoL utility and a cost estimate. Progression is defined by an absolute decline in FVC per cent predicted of $\geq 10 \%$ from a baseline (recently-diagnosed) value, based on the included RCTs. Published sources were used to inform the probability of a lung transplant; survival after lung transplant; and all-cause mortality by age. Acute exacerbations are not modelled as separate health states but are associated with a cost and utility decrement. Model cycle length is one month and a lifetime horizon of 30 years was adopted to capture all clinically and economically important events. A half-cycle correction is applied. Key assumptions are that all patients are in in the unprogressed state initially; those experiencing a $\geq 10 \%$ absolute decline in $\mathrm{FVC} \%$ predicted are considered to be in the progressed health state; and treatment has a constant effect on relative rate of $\mathrm{FVC} \%$ decline, $\mathrm{FVC} \%$ predicted was used as a proxy for disease severity when assigning utilities to the health states. In addition, where treatment costs for any individual treatment were not available an assumed cost was used and tested in threshold analysis.
Treatment effects were obtained from the NMA. Utility values from the systematic review of HRQoL are applied to the modelled health states to estimate the benefits measured as quality adjusted life years (QALYs). Costs are included for treatments, treatment monitoring, acute exacerbations, lung transplant and adverse events, based on the UK health system. Future costs and benefits are discounted at $3.5 \%$ per annum. The outcome is reported as cost per QALY gained against the next best alternative treatment using incremental cost effectiveness ratios (ICERs). The model examines uncertainty in deterministic and probabilistic sensitivity analyses. Model validation was undertaken by checking structure, calculations and data inputs. In addition the advisory group reviewed the structure and internal consistency was examined by varying input values. Model results were compared with trial outputs and other publications [15-17].

\section{Results}

\section{Clinical effectiveness}

Searches identified 905 unique references and 64 of these were retrieved after screening of titles and abstracts. Fourteen studies (13 RCTs and 1 CCT) were included (Figure 1). Four RCTs evaluated the use of pirfenidone, $[15,18,19]$ three the use of n-acetylcysteine (alone or in combination), [20-22] one azathioprine, [23] one nintedanib, [16] one sildenafil, [24] one thalidomide, [25] one a pulmonary rehabilitation programme, [26] and one a disease management programme [27]. In addition one CCT of pulmonary rehabilitation was included [28]. This study was published in Polish and translation of key methods and results only were undertaken due to resource and time constraints. Therefore caution is recommended in interpreting our assessment of this study. No studies of palliative care interventions were identified that met the inclusion criteria. Study quality was generally good with a low risk of bias. Ten studies were undertaken in populations that would likely be classed as mild to moderate IPF [29]. The majority of these studies had reasonable sample sizes and duration of follow was between nine months and 16 months. Four studies were undertaken in populations that would be classed as moderate to severe IPF. Three of these were the non-pharmacological intervention studies, and one the drug sildenafil. Sample sizes were generally smaller in the non-pharmacological studies, and there was no long-term follow up. Across all studies the mean ages of participants ranged from approximately 54-69 years, the gender ratio of males to females was generally $3: 1$, and the duration of diagnosis tended to be between 1 and 3 years. In the ten studies in mild-to-moderate IPF the baseline FVC ranged between $65-90 \%$ and in the four studies in moderate-tosevere IPF this ranged from $55-70 \%$. The populations were deemed to be reasonably similar to those seen in clinical 


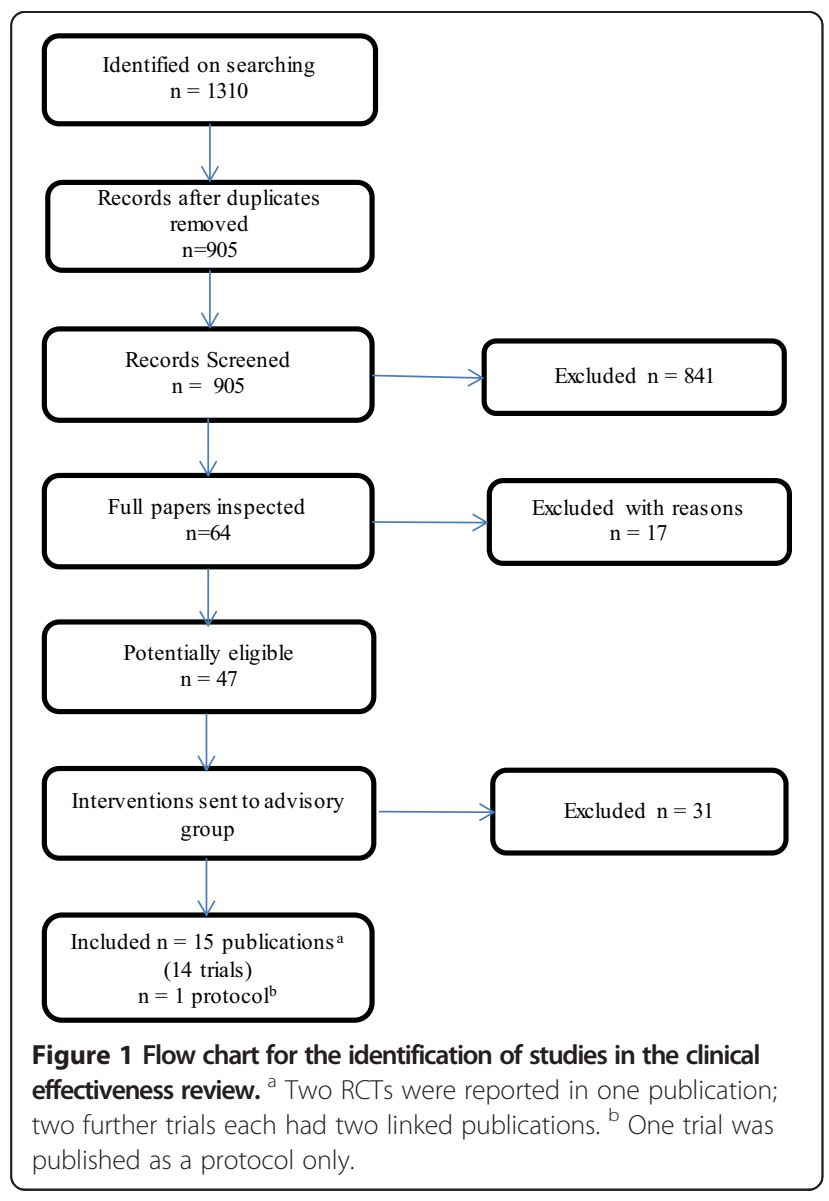

practice by our advisory group. For further summary details see Table 1.

Results for the clinical effectiveness of the five pharmacological interventions in patients with mild to moderate IPF were mixed. In clinical practice azathioprine is only used in restricted circumstances, in one RCT [23] azathioprine and prednisolone led to an improvement in survival compared with placebo and prednisolone when an age adjusted analysis was used. There was no effect on lung function. This trial had an unclear risk of bias, a small sample size, and it is uncertain whether all patients had a diagnosis of IPF based on current recommendations. Consequently, caution is recommended when interpreting results. Nintedanib $300 \mathrm{mg} /$ day was more favourable than placebo on some FVC measures, acute exacerbations and mortality, however, the primary outcome of annual rate of decline in FVC was not statistically significant [16]. Treatment with NAC was evaluated in three studies, [20-22] in combination with azathioprine and prednisolone in two (triple therapy) and in an inhaled format in one. Study results were mixed and establishing the stand-alone effect of NAC is difficult due to the differences between the three studies. There was no benefit from triple therapy on FVC compared to placebo in one trial, however, there was a benefit on vital capacity (VC) when compared to azathioprine and prednisolone in another. The treatment effect of inhaled NAC was not statistically different from that of a control group $(p=0.05)$. The study using inhaled NAC had an unclear risk of bias which should be considered when interpreting results. Four RCTs $[15,18,19]$ evaluated the use of pirfenidone and meta-analysis shows that pirfenidone appears to demonstrate a significant effect on FVC when compared to placebo (SMD 0.24, 95\% CI 0.06, 0.41, $\mathrm{p}=$ 0.008 ). This should be cautiously interpreted as the outcomes pooled were different, the timing of assessment varied (from 48 weeks to 72 weeks) and there was moderate statistical heterogeneity $\left(\mathrm{I}^{2}=45 \%\right)$. The effect of pirfenidone on secondary outcomes was more uncertain. Thalidomide was assessed in those with cough in a small crossover RCT [25]. HRQoL outcomes related to cough were improved with thalidomide compared to placebo. There is no evidence relating to any subgroups in any of the published studies.

One study [24] assessed sildenafil for those with moderate to severe IPF. No statistically significant benefit of sildenafil was seen on the primary outcome, a $20 \%$ improvement on the six minute walk test. Results on secondary outcomes were mixed with some favourable to sildenafil but others being not statistically significant.

Adverse events were generally mild to moderate and reasonably well balanced between the treatments and controls with the exception of thalidomide which led to a greater proportion of people experiencing at least one adverse event $(77 \%)$ than the placebo treated participants (22\%) [25]. Severe adverse events appeared to be more common in one study in those treated with triple therapy [21].

Ten studies of pharmacological interventions were included in NMA; the resulting evidence network is shown in Figure 2. Thalidomide was excluded as the focus of treatment is not on lung function. Inhaled NAC was considered separately from triple therapy owing to its different method of administration. Both direct and indirect evidence was used to assess the treatment effect compared to placebo. Only the fixed effect results for nintedanib and pirfenidone were statistically significant, odds ratios for reducing the rate of decline in FVC compared to placebo are shown in (Table 2). The random effects model did not demonstrate a better fit than the fixed effect model and there was no evidence of inconsistency within the evidence network. A head-to-head comparison of nintedanib versus pirfenidone suggested a trend favouring nintedanib, but this was not statistically significant and should be cautiously interpreted in the light of the various differences between the studies (Table 2). Further trial evidence could be used to test this further.

Three non-pharmacological intervention studies compared a pulmonary rehabilitation programme or disease management programme to control interventions in moderate-to-severe IPF. Results from the two pulmonary 
Table 1 Study characteristics of included interventions

\begin{tabular}{|c|c|c|c|}
\hline $\begin{array}{l}\text { Study and intervention } \\
\text { details }\end{array}$ & Baseline characteristics & Outcomes & $\begin{array}{l}\text { Risk of bias } \\
\text { (selection bias) }\end{array}$ \\
\hline \multicolumn{4}{|l|}{ Pharmacological agents } \\
\hline \multicolumn{4}{|l|}{ Azathioprine } \\
\hline Raghu et al. 1991 [21] & Mean Age: 56 years & \multirow{4}{*}{$\begin{array}{l}\text { Primary outcomes: not stated } \\
\text { as primary or secondary: } \\
\text { measurable change in lung } \\
\text { function (FVC, DLCO, P[A-a]O2) } \\
\text { at } 12 \text { months; survival }\end{array}$} & \multirow[t]{8}{*}{ Unclear risk } \\
\hline Country: USA & M/F\%: 55/45 & & \\
\hline Design: RCT & Time since diagnosis: 2 years & & \\
\hline Number of centres: 2 & FVC: $67 \%$ & & \\
\hline $\begin{array}{l}\text { Funding: Grant from Virginia Mason ResearchCentre, } \\
\text { Seattle, USA }\end{array}$ & & \multirow[t]{4}{*}{ Length of follow-up: 12 months } & \\
\hline \multicolumn{2}{|l|}{ Interventions: } & & \\
\hline \multicolumn{2}{|l|}{ 1. Prednisone and placebo, $n=13$} & & \\
\hline 2. Prednisone and azathioprine, $n=14$ & & & \\
\hline
\end{tabular}

Duration of treatment: 12 months

\section{BIBF-1120}

Richeldi et al. 2011 [22]

Country: 25 countries including Italy, Mexico,

Mean Age: 65 years

Primary outcomes: annual rate of decline in FVC

Low risk

M/F\%: 75/25

Germany, USA, Korea, UK, France.

Design: RCT (dose finding phase II study)

Time since diagnosis: 1.2 Secondary outcomes: \% predicted

Number of centres: 92 years FVC; DLCO; SpO2; TLC; 6MWT, $S G R Q$, decrease in FVC of more than $10 \%$ or more than $200 \mathrm{ml}$; SpO2 decrease of more than $4 \%$; acute exacerbations; survival; death from a respiratory cause; adverse events

Funding: supported by Boehringer Ingelheim

Interventions:

1. BIBF $112050 \mathrm{mg} /$ day, $\mathrm{n}=86$

2. BIBF $112050 \mathrm{mg}$ twice per day ( $100 \mathrm{mg} /$ day), $\mathrm{n}=86$

Length of follow-up: 54 weeks

3. BIBF $1120100 \mathrm{mg}$ twice per day (200 mg/day), $n=86$

4. BIBF $1120150 \mathrm{mg}$ twice per day (300 mg/day), $n=85$

5. Placebo, $n=85$

Duration of treatment: 52 weeks

\section{$\mathrm{N}$-Acetylcysteine (alone or in combination)}

Demedts et al. 2005 [18]

Country: Belgium, France, Germany, Italy, Spain, the Netherlands

Design: RCT

FVC: $80 \%$

Number of centres: 36

Funding: sponsored by the Zambon group

Interventions:

1. N-acetylcysteine, prednisolone, azathioprine, $n=92$

(80 analysed)

2. Placebo, prednisolone, azathioprine, $n=90$ (75 analysed)

Duration of treatment: not stated, assume 12 months.
Mean Age: 63 years

Primary outcomes: absolute changes in VC and DLCO at 12 months

M/F\%: 72/28

Time since diagnosis: 1.6 years

FVC: $66 \%$
Secondary outcomes: \% predicted VC, \% predicted DLCO, alveolar volume change and \% predicted, CRP score, dyspnoea score, maximum exercise indexes, HRCT outcomes, SGRQ, adverse events, withdrawals, and mortality

Raghu et al., (IPFCRN) 2012 [19] Mean Age: 68 years

Length of follow-up: 12 months 
Table 1 Study characteristics of included interventions (Continued)

Country: USA
Design: RCT (PANTHER study)
Number of centres: 25
Funding: grants from the NHLBl; the Cowlin Family fund
NAC and placebo donated by Zambon
Interventions:
1. N-acetylcysteine and placebo (data not presented
in article as 'ongoing' data collection), $\mathrm{n}=81$
2. N-acetylcysteine/prednisolone/azathioprine, $\mathrm{n}=77$
3. Placebo, $\mathrm{n}=78$
Duration of treatment: up to 60 weeks

\begin{tabular}{ll}
\hline Homma et al. $2012[20]$ & Mean Age: 68 years \\
Country: Japan & M/F\%: $76 / 24$ \\
Design: RCT & Time since diagnosis: \\
& 3 years \\
Number of centres: 27 & FVC: $89 \%$
\end{tabular}

Funding: grant from Ministry of Health, Labour and Welfare

Interventions:

1. N-acetylcysteine inhaled, $\mathrm{n}=51$ (38 analysed)

2. Control, $n=49$ (38 analysed) Duration of treatment: 48 weeks

M/F\%: 75/25

Time since diagnosis: 1 year Secondary outcomes: rate of death,

FVC: $71 \%$ time until death, frequency of acute exacerbation, frequency of maintained FVC response, time to disease progression, clinical and physiological measures including: DLCO, 6MWT, CPI, UCSD SBQ, SGRQ,

SF-36, EQ-5D. Adverse events.

Length of follow-up: 60 weeks in the planned analysis. The study was stopped early. The mean follow-up was 32 weeks.

Primary outcomes: absolute change in FVC at 48 weeks

Unclear risk

Secondary outcomes: changes in lowest aterial $\mathrm{O} 2$ saturation, $6 \mathrm{MWT}$ distance, PFT parameters $\mathrm{VC}, \%$ predicted VC, TLC, \% predicted TLC, DLCO, predicted DLCO), serum markers of pneumocyte injury; disease progression as determined by HRCT; subjective changes in symptoms such as dyspnoea, adverse events.

Length of follow-up: 48 weeks

\section{Pirfenidone}

Noble et al., 2011 [15]

Mean Age: 67 years

Primary outcomes: change in per cent predicted FVC

Capacity study 006

M/F\%: 72/28

Time since diagnosis: $\leq 1$

year: $59 \%$

Secondary outcomes: categorical FVC (5-point scale), progression-free survival, worsening IPF, dyspnoea, 6MWT distance, worst peripheral oxygen saturation (SpO2) during the 6MWT, per cent predicted DLCO, fibrosis, mortality.

Number of centres: 110 centres

Funding: InterMune

Interventions:

1. Pirfenidone $2403 \mathrm{mg} /$ day, $\mathrm{n}=171$

2. Placebo, $n=173$

Length of follow-up: 72 weeks from the date the last patient was enrolled.

Duration of treatment: 72 weeks

Noble et al., 2011 [15]

Mean Age: 66 years

Primary outcomes: change in per cent

Low risk

Capacity study 004

M/F\%: 71/29 predicted FVC

Country: Australia, Belgium, Canada, France, Germany, Ireland, Time since diagnosis: $\leq 1$ Italy, Mexico, Poland, Spain, Switzerland, UK, USA

Secondany outcomes: categorical FVC (5-point scale), progression-free survival, worsening IPF, dyspnoea, 6MWT distance, worst peripheral oxygen saturation (SpO2) during the 6MWT, per cent predicted

Design: RCT

FVC: $75 \%$

Number of centres: 110 centres

Funding: InterMune

Interventions:

1. Piffenidone $2403 \mathrm{mg} / \mathrm{day}, \mathrm{n}=174$

2. Pirfenidone $1197 \mathrm{mg} / \mathrm{day}, \mathrm{n}=87$
DLCO, mortality. 
Table 1 Study characteristics of included interventions (Continued)

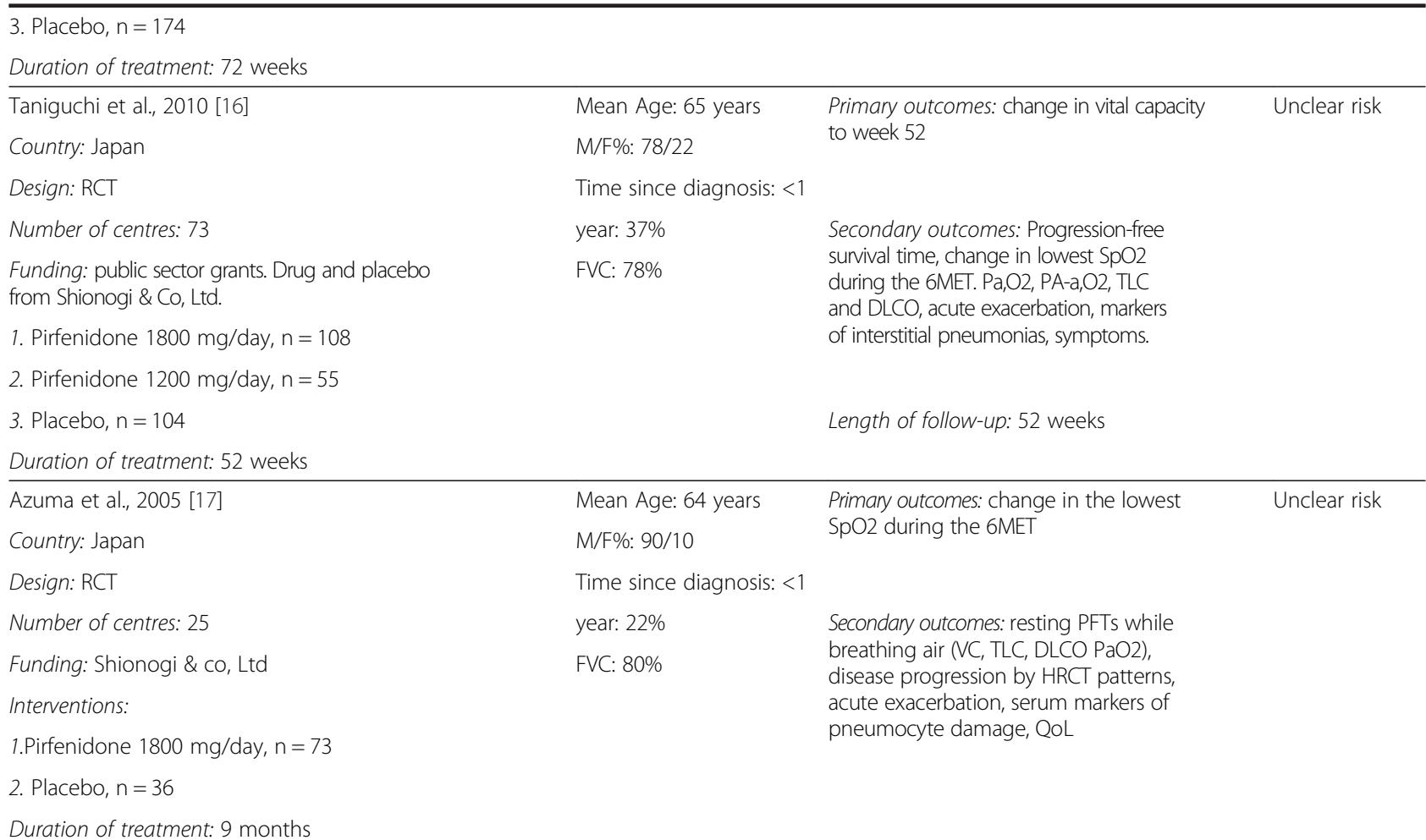

Length of follow-up: minimum of 9 months

\section{Thalidomide}

Horton et al., 2012 [24]

Country: USA

Design: randomised cross-over trial

Number of centres: one

Funding: Celgene Corporation

Interventions:

1. Thalidomide, $n=23$

2. Placebo, $n=23$

Duration of treatment: 12 weeks each treatment with a

2 week washout period between treatments.

$\begin{array}{lll}\text { Mean Age: } 68 \text { years } & \text { Primary outcomes: cough-specific quality } & \text { Low risk } \\ \text { M/F\%: } 78 / 22 & \text { of life (CQLQ) } & \end{array}$

M/F\%: 78/22

Time since diagnosis: 1.7

years

FVC: $70 \%$

Secondary outcomes: cough, respiratory quality of life.

Method of assessing outcome: Cough-specific quality of life measured by CQLQ. Cough measured by $10 \mathrm{~cm}$ VAS. Respiratory quality of life measured by SGRQ.

Length of follow-up: 12 weeks.

\section{Sildenafil (severe IPF)}

Zisman and colleagues IPFCRN, 2010 [23]

Country: USA

Design: RCT

Number of centres: 14

Funding: NHLBl; the Cowlin Fund (Chicago Community trust); FVC: 57\% Pfizer; Masimo

1. Sildenafil, $n=89$

2. Placebo, $\mathrm{n}=91$

Duration of treatment: 12 weeks.

years

Mean Age: 69 years

M/F\%: 84/16

Time since diagnosis: 1.9

Primary outcomes: presence or absence of an improvement of at least $20 \%$ in the 6MWT distance at 12 weeks.

Secondary outcomes: changes in the 6MWT distance, degree of dyspnoea, quality of life, FVC, DLCO, arterial partial pressure of oxygen and arterial oxygen saturation, and the alveolar-arterial oxygen gradient while breathing ambient air, adverse events, hospitalisations, death.

Length of follow-up: 12 weeks 
Table 1 Study characteristics of included interventions (Continued)

\begin{tabular}{|c|c|c|c|}
\hline \multicolumn{4}{|l|}{ Non-pharmacological interventions } \\
\hline \multicolumn{4}{|c|}{ Disease management programme/Pulmonary Rehabilitation } \\
\hline Lindell et al. 2010 [26] & Mean Age: 66 years & \multirow{5}{*}{$\begin{array}{l}\text { Primany outcomes: Not specified as primary } \\
\text { or secondary outcomes. Dyspnoea (UCSDSBQ); } \\
\text { Anxiety (BAl); Depression (BDI-II); Stress (PSS); } \\
\text { QoL (SF-36) }\end{array}$} & \multirow[t]{9}{*}{ Unclear risk } \\
\hline Country: USA & M/F\%: 76/24 & & \\
\hline Design: $\mathrm{RCT}$ & Time since diagnosis: NR & & \\
\hline Number of centres: one & FVC: $>55: 70 \%$ & & \\
\hline Funding: Fairbanks-Horix Foundation & & & \\
\hline Interventions: & & \multirow[t]{4}{*}{ Length of follow-up: Unclear } & \\
\hline $\begin{array}{l}\text { 1. Program to Reduce IPF Symptoms and Improve } \\
\text { Management (PRISIM), } n=10 \text { pairs }\end{array}$ & & & \\
\hline 2. Usual care, $n=11$ pairs & & & \\
\hline Duration of treatment: 6 weeks & & & \\
\hline Jastrzebski et al. 2008 [27] & Mean Age: 56 years & \multirow{7}{*}{$\begin{array}{l}\text { Primary outcomes: not specified as primary } \\
\text { or secondary. Dyspnoea (oxygen cost diagram, } \\
\text { baseline dyspnoea index). QoL (SF-36), 6MWT } \\
\text { (distance, dyspnoea in Borg's scale), maximal } \\
\text { inspiratory pressure, lung function tests } \\
\text { (IC, TLC, VC, FEV1, DLCOSB, DLCONA). }\end{array}$} & \multirow[t]{9}{*}{ High risk } \\
\hline Country: Poland & M/F\%: 64/36 & & \\
\hline Design: CCT & Time since diagnosis: $>2$ & & \\
\hline Number of centres: one & years & & \\
\hline Funding: not translated & FVC: $68 \%$ & & \\
\hline Interventions: & & & \\
\hline 1. Inspiratory muscle training, $n=16$ & & & \\
\hline 2. Control, $n=14$ & & \multirow[t]{2}{*}{ Length of follow-up: 12 weeks } & \\
\hline Duration of treatment: 12 weeks (two six week cycles) & & & \\
\hline Nishiyama et al. 2008 [25] & Mean Age: 66 years & \multirow{4}{*}{$\begin{array}{l}\text { Primary outcomes: not specified as } \\
\text { primary or secondary. Pulmonary } \\
\text { function tests (FVC, FEV1, TLC, PaO2,PaCO2,) } \\
\text { DLCO, 6MWT; BDI; SGRQ }\end{array}$} & \multirow[t]{9}{*}{ Unclear risk } \\
\hline Country: Japan & M/F\%: 76/24 & & \\
\hline Design: $\mathrm{RCT}$ & Time since diagnosis: NR & & \\
\hline Number of centres: one & FVC: $67 \%$ & & \\
\hline Funding: Japanese ministry of health, labor and welfare & & \multirow{5}{*}{$\begin{array}{l}\text { Length of follow-up: } 10 \text { weeks after the start } \\
\text { of the programme. }\end{array}$} & \\
\hline Interventions: & & & \\
\hline $\begin{array}{l}\text { 1. Pulmonary rehabilitation programme (PRP), } \\
\mathrm{n}=15 \text { ( } 13 \text { analysed) }\end{array}$ & & & \\
\hline \multicolumn{2}{|l|}{ 2. Control, $n=15$} & & \\
\hline Duration of treatment: 10 week programme. & & & \\
\hline
\end{tabular}

rehabilitation studies were inconclusive; some outcomes were favourable to pulmonary rehabilitation but not others. The risk of bias was uncertain and endpoints were only assessed immediately after the interventions finished. Limited evidence of the effectiveness of a disease management programme was demonstrated. This study had an uncertain risk of bias and follow-up was immediate.

\section{Cost effectiveness and HRQoL systematic reviews}

One economic evaluation was identified, which examined a testing strategy prior to treatment with triple therapy but did not examine the cost-effectiveness of treatment. The systematic review of HRQoL included 23 studies that examined HRQoL using either a generic preference-based tool (EuroQol five dimensions [EQ-5D], Short Form-36 [SF-36]) or a disease specific instrument (SGRQ) that could be mapped to utility for the economic model. Results varied between the studies, given the different populations under study and the different measures and time points of measurement, however, results generally showed that IPF has an adverse effect on HRQoL which increases with severity.

\section{Cost-effectiveness of pharmacological treatments for IPF}

The baseline risk of disease progression in the unprogressed state was taken from two of the pirfenidone trials included in the systematic review of clinical effectiveness [15] as the population most closely met the model definition of unprogressed IPF and the RCTs had large sample sizes. The probabilities of progression free survival were obtained from the Kaplan Meier survival curve published for the pooled placebo population of two RCTs [15]. 


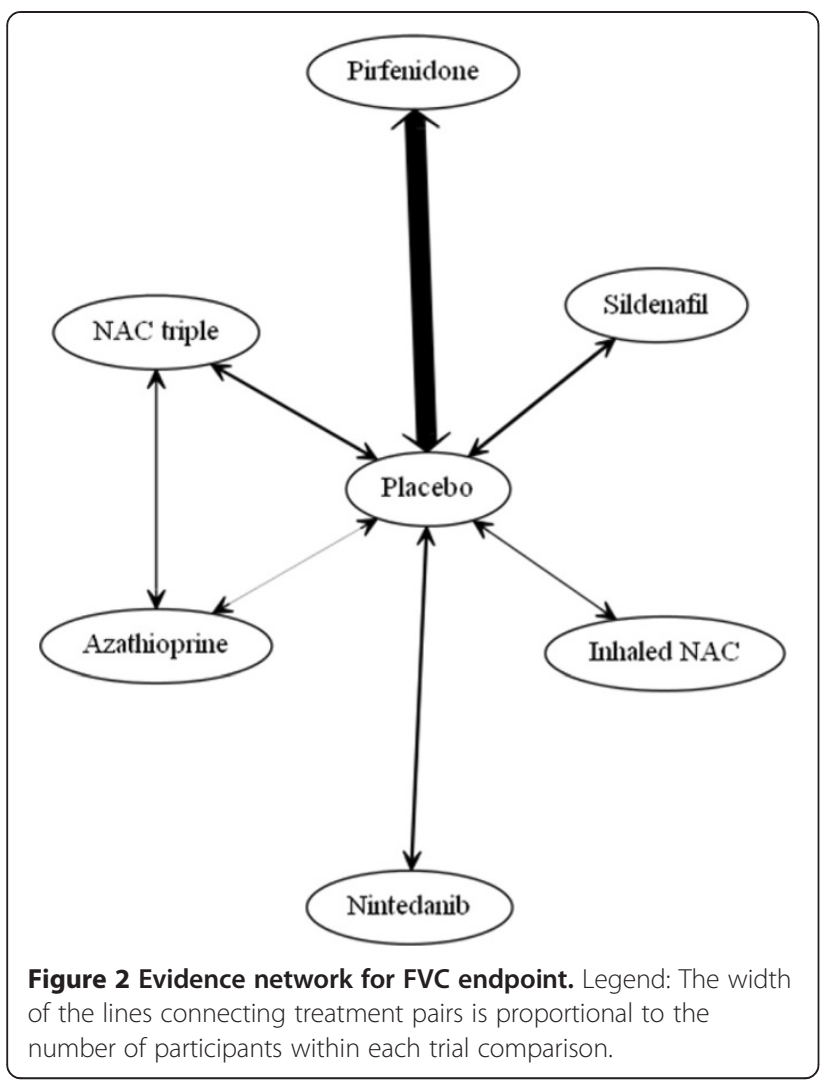

Parametric survival curves were fitted to this curve in Stata using maximum likelihood estimation in order to extrapolate beyond the 72 weeks follow-up. Exponential, Weibull, loglogistic, lognormal and Gompertz parametric models were examined. Goodness of fit was assessed using the Akaike Information Criterion (AIC). The Weibull model was selected because of the balance of good fit (AIC) and face validity (comparison of predicted survival with known survival in IPF). In the progressed state the monthly probability of death was taken from the overall survival curve in a recent study reporting survival for those experiencing a $10 \%$ or greater decline in FVC [30].
This study was an observational study but followed a larger sample than other published studies that differentiate survival by FVC decline and was deemed the most appropriate for the evaluation. Five parametric survival curves were fitted using the distributions noted above. The exponential distribution was selected (using AIC and face validity) to extrapolate beyond the five years of observed data.Probabilities of acute exacerbations in both unprogressed and progressed health states, and probabilities of lung transplant and survival from lung transplant, were taken from published sources.

HRQoL utility values were applied to each of the alive health states. These were not differentiated by treatment; the impact of treatment on utility was assumed to occur because of delay to disease progression which the model accounts for. The HRQoL values used for the unprogressed and progressed IPF health states were taken from EQ-5D values reported in two trials included in the systematic reviews of clinical effectiveness and HRQoL $[21,24]$. These trials were conducted by the same clinical network and it is likely that the estimates of EQ-5D are consistent and can therefore be contrasted in our economic model. The utility values applied in the model can be seen in Table 3. Utility associated with lung transplant is taken from a UK-based study [31] which assessed HRQoL using the EQ-5D. We weighted the utility to account for a greater proportion of single lung transplants in the IPF population than the proportion seen in the study, based on clinical opinion. Utility data for acute exacerbations were not identified in the literature and therefore a utility decrement was applied that was in line with decrements seen in those with asthma and chronic obstructive pulmonary disease (Table 3). Sensitivity analyses were used to test these utility decrements.

Five types of cost are considered in the economic model. The costs associated with each treatment were made up of the costs of the drug and the monitoring costs associated with the treatment. Dose information and unit costs were taken from published sources where available $[32,33]$. Unit

Table 2 NMA Fixed effects results, SMDs converted to log odds ratios for slowing the decline in FVC

\begin{tabular}{|c|c|c|c|c|c|c|c|c|}
\hline \multirow[b]{2}{*}{ Comparator (vs. placebo) } & \multirow[b]{2}{*}{$\mathrm{N}$ studies } & \multirow[b]{2}{*}{ Total N participants } & \multicolumn{3}{|c|}{ Log odds ratios } & \multicolumn{3}{|c|}{ Odds ratios } \\
\hline & & & \multirow{2}{*}{$\begin{array}{c}\text { Mean } \\
0.44\end{array}$} & \multicolumn{2}{|c|}{$95 \% \mathrm{Crl}$} & \multirow{2}{*}{$\begin{array}{c}\text { Mean } \\
1.56\end{array}$} & \multicolumn{2}{|c|}{$95 \% \mathrm{Crl}$} \\
\hline Azathioprine & 1 & 19 & & -0.30 & 1.19 & & 0.74 & 3.29 \\
\hline Nintedanib & 1 & 170 & -0.97 & -1.52 & -0.41 & 0.38 & 0.22 & 0.66 \\
\hline NAC triple therapy & 2 & 294 & -0.06 & -0.60 & 0.48 & 0.94 & 0.55 & 1.62 \\
\hline Inhaled NAC & 1 & 76 & -0.42 & -1.24 & 0.40 & 0.66 & 0.29 & 1.49 \\
\hline Pirfenidone & 4 & 1006 & -0.39 & -0.62 & -0.16 & 0.68 & 0.54 & 0.85 \\
\hline Sildenafil & 1 & 180 & -0.12 & -0.65 & 0.41 & 0.89 & 0.52 & 1.51 \\
\hline \multicolumn{9}{|c|}{ Head-to-head comparison } \\
\hline Nintedanib vs. Pirfenidone & -0.58 & & & -1.18 & 0.03 & 0.56 & 0.31 & 1.03 \\
\hline
\end{tabular}


Table 3 EQ-5D utility values by model health state

\begin{tabular}{ll}
\hline Model health state & EQ-5D (SD) \\
\hline Unprogressed IPF (corresponds with an FVC 72\%) & $0.80(0.20)$ \\
Progressed IPF (corresponds with an FVC 59\%) & $0.74(0.19)$ \\
Lung transplant & \\
0-6 months after transplant & $0.71(0.38)$ \\
7-18 months after transplant & $0.72(0.31)$ \\
19-36 months after transplant & $0.70(0.33)$ \\
$>36$ months after transplant & $0.68(0.38)$ \\
Acute exacerbation decrement & 0.20 (not available) \\
\hline
\end{tabular}

costs and data sources were unavailable for nintedanib and an assumed cost was used which was also subject to full sensitivity analysis. Hospital admission costs arising from acute exacerbations were estimated from NHS reference costs for all treatments as no treatment-specific acute exacerbation costs were available (£1361.04) [34]. Ongoing non-pharmacological treatment costs for management of the condition were included and covered annual home oxygen costs (£824.30) and the costs of long-term oxygen monitoring (£173.94) for the progressed IPF state. The costs associated with lung transplant were calculated using NHS reference costs [34] excluding outpatient procedures $(£ 35,468.61)$. Costs of adverse events were attributed to the pharmacological interventions. Based on the incidence of serious adverse events seen in the trials included in the systematic review of clinical effectiveness, costs were applied for each event per patient in the first cycle of the model [34].

The model base-case results show increased survival for five of the treatments compared with BSC, at increased cost (see Table 4). The combination of azathioprine and prednisolone is dominated by BSC (treatment is more costly and less effective than BSC). Triple therapy is associated with an ICER of $£ 41,811$ per QALY gained when compared to BSC. Inhaled NAC is associated with an ICER of $£ 5,037$ per QALY gained when compared to BSC. Sildenafil, pirfenidone and nintedanib are not cost-effective at a willingness to pay (WTP) threshold of $£ 30,000 / \mathrm{QALY}$ when compared to BSC. Therefore only one treatment, inhaled NAC, is costeffective at a £30,000 WTP threshold, but its treatment effect is not statistically significant in the RCT, a small study with undetermined risk of bias.

Deterministic and probabilistic sensitivity analyses tested uncertainty in model parameter values, including costs and probabilities of acute exacerbation and lung transplant. Treatment effects and utilities were also varied in sensitivity analyses. The parameters were varied between the $2.5^{\text {th }}$ and $97.5^{\text {th }}$ percentiles of the mean value and analyses found that results were generally robust but were particularly sensitive to changes in the value of the treatment effect parameters.
The monthly cost of nintedanib was assumed $(£ 3,274)$. Results demonstrate that, given a WTP of $£ 30,000$ per QALY, nintedanib must cost less than $£ 736$ per month to be considered as the cost-effective treatment option compared to BSC and pirfenidone.

\section{Discussion}

We systematically reviewed evidence for the clinical effectiveness of six pharmacological interventions and two nonpharmacological interventions for IPF. Participants in most of these studies would likely be classed as having mild-tomoderate IPF and generally were similar to those seen in clinical practice. There was a range of treatments under investigation in these trials with only one treatment, pirfenidone, providing evidence from more than one trial that was suitable for a formal meta-analysis to be undertaken. The outcomes reported in these studies differed however, and therefore caution is required when considering the results of the meta-analysis and the narrative synthesis of each of the included studies. In a network meta-analysis of the pharmacological treatments only pirfenidone and nintedanib had a statistically significant treatment effect, reducing the rate of decline in FVC compared to placebo. One pharmacological treatment was excluded from the network meta-analysis as the focus of treatment was not on lung function but on the symptom cough. In this study thalidomide appeared to improve cough, and quality of life compared with a placebo treatment. Results of three studies investigating two non-pharmacological treatments show mixed results and it is therefore unclear how effective these interventions are. There are differences between the studies in terms of the interventions, the participants, and the outcomes reported together with study design issues (e.g. short follow-up) and uncertain risk of bias that may account for some of the results seen.

Evidence from systematic reviews of cost effectiveness and HRQoL identified one economic evaluation of limited relevance and 23 HRQoL studies. These latter studies varied in their populations and study methods but generally

Table 4 Summary of base case results

\begin{tabular}{|c|c|c|c|c|}
\hline Treatment & $\begin{array}{l}\text { Total } \\
\text { costs }(£)\end{array}$ & $\begin{array}{l}\text { Total } \\
\text { QALYs }\end{array}$ & $\begin{array}{l}\text { ICER vs. BSC } \\
\text { (£/QALY) }\end{array}$ & $\begin{array}{l}\text { ICER vs. next best } \\
\text { option ( } £ / Q A L Y)\end{array}$ \\
\hline BSC & 3,084 & 2.98 & - & - \\
\hline $\begin{array}{l}\text { Azathioprine } \\
\text { \& prednisolone }\end{array}$ & 4,313 & 2.66 & Dominated & Dominated \\
\hline $\begin{array}{l}\text { NAC triple } \\
\text { therapy }\end{array}$ & 5,021 & 3.03 & 41,811 & $\begin{array}{l}\text { Extended } \\
\text { Dominance }\end{array}$ \\
\hline Inhaled NAC & 5,029 & 3.37 & 5,037 & 5,037 \\
\hline Sildenafil & 12,008 & 3.11 & 68,116 & Dominated \\
\hline Pirfenidone & 70,118 & 3.34 & 190,146 & Dominated \\
\hline Nintedanib & 139,613 & 4.01 & 132,658 & 209,246 \\
\hline
\end{tabular}

NB: Nintedanib uses an assumed cost. 
showed that IPF has an adverse effect on HRQoL compared to population norms, and that HRQoL diminishes as IPF progresses. A new decision analytic health economic model was developed to assess the cost-effectiveness of six pharmacological treatments for IPF. Results show increased survival for five of the treatments compared with best supportive care, at increased cost. Only one treatment, inhaled NAC, is cost-effective at a WTP threshold of $£ 30,000$ but no statistically significant treatment effect was seen in the RCT or our NMA. Probabilistic sensitivity analysis showed that inhaled NAC has a $65 \%$ probability of being cost-effective if a decision threshold of $£ 20,000$ per QALY gained is used. Although pirfenidone and nintedanib achieve a statistically significant treatment effect in NMA they each have a probability of $0 \%$ of being cost-effective at a threshold of $£ 30,000$ per QALY. This is based on an assumed cost for nintedanib. A sensitivity analysis indicated that nintedanib must cost less than $£ 736$ per month to be considered cost-effective.

The past few years have seen an increasing interest in the management of IPF, with pharmacological companies evaluating a range of potential interventions, and a number of influential bodies producing guidelines. However, this systematic review demonstrates that at present there are few treatments which have any effect on surrogate outcomes which can be linked through evidence to patient related outcomes such as mortality. The findings of our research also suggest that under current willingness to pay thresholds only one treatment is likely to be cost effective, although, general recommendations cannot be made due to limitations in the evidence base. In terms of a cure it is considered that lung transplantation is the only intervention available which has curative intent. However, no evidence on lung transplant was eligible for inclusion in this evidence synthesis and so this could not be evaluated formally. There is also a scarcity of studies on interventions for symptom management and palliative care in IPF despite this being a recommended approach in recent clinical guidance [5].

No previous systematic reviews have included all potentially relevant treatments for IPF, and there has only been limited economic evaluation previously. Our results are useful to clinicians and patients, and complement recent national guidance by NICE in the UK [5]. In addition to standard synthesis we undertook a network meta-analysis to compare the pharmacological therapies to a common comparator. Our results show that only two treatments (nintedanib and pirfenidone) significantly slow the decline in FVC compared to placebo under a fixed effect model. However, with few studies it was not possible to fully explore heterogeneity within these data and the results should be cautiously interpreted. We also undertook an illustrative analysis comparing nintedanib with pirfenidone through an indirect comparison. While this showed a trend favouring nintedanib, it was not statistically significant and should be interpreted with caution until such time that a more complete analysis can be undertaken on more robust data. We identified a number of ongoing trials of potential relevance. Our evidence synthesis has highlighted the current evidence base in which these new trials can be contextualised once they report.

There were numerous differences between the studies included in this review. However we applied a rigorous approach to the inclusion, quality assessment and data synthesis of the studies (laid out in a research protocol), to ensure that our work was as unbiased as possible. Our research was guided by an advisory group from its initiation, in particular to ensure that the interventions included were appropriate to current or future management in the NHS. We ensured that only the highest quality studies were included to limit uncertainty in the results.

Our study has several limitations. The meta-analysis and NMA used the standardised mean difference to express findings from studies on a common scale. In this case we combined mean change in $\mathrm{FVC} \%$ predicted with absolute change in FVC, albeit the former is adjusted for certain baseline characteristics, and this should be considered when interpreting the results. Many of the included studies compared treatments to placebo. Few direct comparisons were identified and results of an indirect comparison via the NMA approach are presented. However there are known limitations to the use of indirect comparisons which should also be considered in interpreting our findings [35]. The economic model assumes that treatments have a constant effect on the relative rate of $\mathrm{FVC} \%$ decline compared to $\mathrm{BSC}$, but treatment may in fact become less effective with time or as the condition progresses. (This would make the treatments less costeffective than shown in our results.) Finally, because of limitations in the data the absolute decline in $\mathrm{FVC} \%$ predicted was used as the measure of disease progression in the model. It is possible that use of this measure may introduce bias because the starting FVCs of patients (which might vary widely) is not taken into account.

\section{Conclusions}

This evidence synthesis reports on the effectiveness of a range of interventions for IPF and complements recent UK guidance [5]. The current evidence suggests that there are currently few treatments which are clinically and costeffective. Pirfenidone and nintedanib offer the potential for hope to sufferers and their clinicians, however, their cost-effectiveness is likely to be prohibitive. This research has thoroughly examined the current evidence and can be seen as a platform from which the clinical importance of newer treatments can be assessed when ongoing trials report. The systematic review has highlighted the need for further research into interventions to help alleviate or 
control symptoms of this debilitating condition, in particular pulmonary rehabilitation programmes and thalidomide. Given the results of our study and the weaknesses of the inhaled NAC trial, a well-designed RCT of inhaled NAC should also be considered; our search of ongoing RCTs failed to identify any such studies currently underway.

\section{Additional file}

Additional file 1: NMA model code. Random effects model code for the NMA.

\begin{abstract}
Abbreviations
BSC: Best supportive care; CCT: Controlled clinical trial; EQ-5D: EuroQol five dimensions; FVC: Forced vital capacity; HRQoL: Health related quality of life; ICER: Incremental cost-effectiveness ratio; IPF: Idiopathic pulmonary fibrosis; UK: United Kingdom; NAC: N-acetylcysteine; NHS: National health service; NICE: National institute for health and care excellence; NMA: Network meta-analysis; QALY: Quality adjusted life Year; RCT: Randomised controlled trial; SD: Standard deviation; SF-36: Short Form-36; SMD: Standardised mean difference; VC: Vital capacity; WTP: Willingness to pay.
\end{abstract}

\section{Competing interests}

KOR and AW have previously been members of the Intermune advisory board for pirfenidone and received payment for research travel expenses only. All authors have completed the unified competing interest form at www.icmje.org/coi_disclosure.pdf (available on request from the corresponding author) and declare 1) no financial support for the submitted work from anyone other than their employer; 2) no financial relationships with commercial entities that might have an interest in the submitted work 3) no spouses, partners, or children with relationships with commercial entities that might have an interest in the submitted work; and 4) no non-financial interests that may be relevant to the submitted work.

\section{Authors' contributions}

EL (Senior Research Fellow) developed the original research application, developed the research protocol, contributed to the background section, assisted in the development of the search strategy, assessed studies for inclusion, extracted data from and quality assessed included studies, synthesised evidence, drafted and edited the final report, project managed the study and is the guarantor of the study; VRC (Senior Research Fellow, Health Economics) assessed studies for inclusion, extracted data from and quality assessed included studies, synthesised evidence, developed the economic evaluation, drafted the report; JLC (Senior Research Fellow) developed the original research application, contributed to the development of the research protocol, contributed to the background section, assessed studies for inclusion, extracted data from and quality assessed included studies, synthesised evidence and drafted the report; DAS (Senior Director, Health Economics, Icon PLC) contributed to the development of the research protocol, synthesised evidence, assisted in developing the economic evaluation, drafted the report; AJC (Professor/Director of SHTAC) developed the original research application, contributed to developing the research protocol, extracted data from and quality assessed included studies, synthesised evidence, drafted the report, provided quality assurance for the project; JJ (Principal Research Fellow, Health Economics) developed the original research application, developed the research protocol, assisted in developing the economic evaluation, drafted the report; KMAO (Consultant Physician) drafted the background section, assisted with the interpretation of the clinical effectiveness evidence, provided clinical advice to the project, drafted the report; SS (Professor of Pulmonary and Cardiac Rehabilitation) drafted the background section, assisted with the interpretation of the clinical effectiveness evidence, provided expert advice to the project, drafted the report; $C B$ (Professor of Palliative Medicine) drafted the background section, assisted with the interpretation of the clinical effectiveness evidence, provided expert advice to the project, drafted the report; AW (Professor/ Consultant Physician) assisted with the interpretation of the clinical effectiveness evidence, provided expert advice to the project, drafted the report. All authors read and approved the final manuscript.

\section{Acknowledgements}

We would like to thank members of our advisory group who provided expert advice and comments on the protocol and the interpretation of the findings of this research. We are also grateful to Karen Welch, Information Specialist, SHTAC, University of Southampton, for generating and running the literature searches, Stuart Mealing, Health Economics, Oxford Outcomes for technical assistance with the economic model, Fiona Boyle, Finance Manager, University Hospitals Southampton Foundation Trust, for sourcing information on relevant costs for the economic evaluation, Helen Thomas, NHS Blood and Transplant for assistance with data related to lung transplantation, and Jonathan Shepherd, Principal Research Fellow, SHTAC, University of Southampton, for reviewing a draft of the full report that this paper is taken from.

This project was funded by the National Institute for Health Research Health Technology Assessment (NIHR HTA) programme. It will be published in full in the Health Technology Assessment journal series (www.nets.nihr.ac.uk/ programmes/hta) in late 2014. The views and opinions expressed therein are those of the authors and do not necessarily reflect those of the HTA programme, NIHR, NHS or the Department of Health.

\section{Author details}

'Southampton Health Technology Assessments Centre (SHTAC), University of Southampton, Southampton, UK. ${ }^{2}$ Icon PLC, Oxford, UK. ${ }^{3}$ Mater Misericordiae University Hospital, Dublin, Ireland. ${ }^{4}$ University Hospitals of Leicester NHS Trust, Leicester, UK. ${ }^{5}$ Department of Palliative Medicine, University Hospital of Munich, Munich, Germany. ${ }^{6}$ Green Lane Hospital, London, UK.

Received: 23 January 2014 Accepted: 4 November 2014

Published: 19 November 2014

\section{References}

1. Swigris JJ, Stewart AL, Gould MK, Wilson SR: Patients' perspectives on how idiopathic pulmonary fibrosis affects the quality of their lives. Health Qual Life Outcomes 2005, 3:61.

2. Meltzer EB, Noble PW: Idiopathic pulmonary fibrosis. Orphanet J Rare Dis 2008, 3:8. www.ojrd.com/content/3/1/8

3. Navaratnam V, Fleming KM, West J, Smith CJ, Jenkins RG, Fogarty A Hubbard RB: The rising incidence of idiopathic pulmonary fibrosis in the U.K. Thorax 2011, 66:462-467.

4. Raghu G, Weycker D, Edelsberg J, Bradford WZ, Oster G: Incidence and prevalence of idiopathic pulmonary fibrosis. Am J Respir Crit Care Med 2006, 174:810-816.

5. NICE: Diagnosis and management of suspected idiopathic pulmonary fibrosis CG 163. London: NICE; 2013.

6. Raghu G, Collard HR, Egan JJ, Martinez FJ, Behr J, Brown KK, Colby TV, Cordier JF, Flaherty KR, Lasky JA, Lynch DA, Ryu JH, Swigris JJ, Wells AU, Ancochea J, Bouros D, Carvalho C, Costabel U, Ebina M, Hansell DM, Johkoh T, Kim DS, King TE Jr, Kondoh Y, Myers J, Muller NL, Nicholson AG, Richeldi L, Selman M, Dudden RF, et al: An official ATS/ERS/JRS/ ALAT statement: idiopathic pulmonary fibrosis: evidence-based guidelines for diagnosis and management. Am J Respir Crit Care Med 2011, 183:788-824.

7. Christie JD, Edwards LB, Kucheryavaya AY, Benden C, Dipchand Al, Dobbels F, Kirk R, Rahmel AO, Stehlik J, Hertz MI: The registry of the international society for heart and lung transplantation: 29th adult lung and heart-lung transplant report-2012. J Heart Lung Transplant 2013, 31:1073.

8. Centre for Reviews and Dissemination: Systematic reviews: CRD's quidance for undertaking reviews in health care. Third edition. York: York Publishing Services Ltd., CRD; 2009.

9. Drummond MF, Jefferson TO: Guidelines for authors and peer reviewers of economic submissions to the BMJ. The BMJ economic evaluation working party. BMJ 1996, 313:275-283.

10. Philips Z, Ginnelly L, Sculpher M, Claxton K, Golder S, Riemsma R, Woolacoot N, Glanville J: Review of guidelines for good practice in decision-analytic modelling in health technology assessment. Health Technol Assess 2004 8:1-158.

11. Higgins JP, Green S: Cochrane handbook for systematic reviews of interventions version 5.1.0 [updated march 2011]. Cochrane Collaboration 2011, Available from www.cochrane-handbook.org.

12. Caldwell D, Ades AE, Higgins JPT: Simultaneous comparison of multiple treatments: combining direct and indirect evidence. BMJ 2005, 331:897-900. 
13. Dias S, Welton NJ, Sutton AJ, Ades AE: NICE DSU technical support document 2: a generalised linear modelling framework for pairwise and network meta-analysis of randomised controlled trials. 2011. http://www.nicedsu.org.uk .14-6-0013.

14. Chinn S: A simple method for converting an odds ratio to effect size for use in meta-analysis. Stat Med 2000, 19:3127-3131.

15. Noble PW, Albera C, Bradford WZ, Costabel U, Glassberg MK, Kardatzke D, King TE Jr, Lancaster L, Sahn SA, Szwarcberg J, Valeyre D, du Bois RM: Pirfenidone in patients with idiopathic pulmonary fibrosis (CAPACITY): two randomised trials. Lancet 2011, 377:1760-1769.

16. Richeldi L, Costabel U, Selman M, Kim DS, Hansell DM, Nicholson AG, Brown KK, Flaherty KR, Noble PW, Raghu G, Brun M, Gupta A, Juhel N, Kluglich M, du Bois RM: Efficacy of a tyrosine kinase inhibitor in idiopathic pulmonary fibrosis. N Engl J Med 2011, 365:1079-1087.

17. Hagaman JT, Kinder BW, Eckman MH: Thiopurine S-methyltranferase testing in idiopathic pulmonary fibrosis: a pharmacogenetic costeffectiveness analysis. Lung 2010, 188:125-132.

18. Taniguchi H, Ebina M, Kondoh Y, Ogura T, Azuma A, Suga M, Taguchi Y, Takahashi H, Nakata K, Sato A, Takeuchi M, Raghu G, Kudoh S, Nukiwa T: Pirfenidone in idiopathic pulmonary fibrosis. Eur Respir J 2010, 35:821-829.

19. Azuma A, Nukiwa T, Tsuboi E, Suga M, Abe S, Nakata K, Taguchi Y, Nagai S, Itoh H, Ohi M, Sato A, Kudoh S: Double-blind, placebo-controlled trial of pirfenidone in patients with idiopathic pulmonary fibrosis. Am J Respir Crit Care Med 2005, 171:1040-1047.

20. Demedts M, Behr J, Buhl R, Costabel U, Dekhuijzen R, Jansen HM, MacNee W, Thomeer M, Wallaert B, Laurent F, Nicholson AG, Verbeken EK, Verschakelen J, Flower CD, Capron F, Petruzzelli S, De Vuyst P, van den Bosch JM, Rodriguez-Becerra E, Corvasce G, Lankhorst I, Sardina M, Montanari M: High-dose acetylcysteine in idiopathic pulmonary fibrosis. N Engl J Med 2005, 353:2229-2242.

21. Raghu G, Anstrom KJ, King J, Lasky JA, Martinez FJ: Prednisone, azathioprine, and N-acetylcysteine for pulmonary fibrosis. N Engl J Med 2012, 366:1968-1977.

22. Homma S, Azuma A, Taniguchi H, Ogura T, Mochiduki Y, Sugiyama Y, Nakata K, Yoshimura K, Takeuchi M, Kudoh S: Efficacy of inhaled $\mathrm{N}$-acetylcysteine monotherapy in patients with early stage idiopathic pulmonary fibrosis. Respirology 2012, 17:467-477.

23. Raghu G, Depaso WJ, Cain K, Hammar SP, Wetzel CE, Dreis DF, Hutchinson J, Pardee NE, Winterbauer $\mathrm{RH}$ : Azathioprine combined with prednisone in the treatment of idiopathic pulmonary fibrosis: a prospective double-blind, randomized, placebo-controlled clinical trial. Am Rev Respir Dis 1991, 144:291-296.

24. Zisman DA, Schwarz M, Anstrom KJ, Collard HR, Flaherty KR, Hunninghake GW: A controlled trial of sildenafil in advanced idiopathic pulmonary fibrosis. N Engl J Med 2010, 363:620-628.

25. Horton MR, Santopietro V, Matthew L, Horton KM, Polito AJ, Liu MC, Danoff SK, Lechtzin N: Thalidomide for the treatment of cough in idiopathic pulmonary fibrosis. Ann Intern Med 2012, 157:398-406.

26. Nishiyama O, Kondoh Y, Kimura T, Kato K, Kataoka K, Ogawa T, Watanabe F, Arizono S, Nishimura K, Taniguchi H: Effects of pulmonary rehabilitation in patients with idiopathic pulmonary fibrosis. Respirology 2008, 13:394-399.

27. Lindell KO, Olshansky E, Song MK, Zullo TG, Gibson KF, Kaminski N, Hoffman LA: Impact of a disease-management program on symptom burden and health-related quality of life in patients with idiopathic pulmonary fibrosis and their care partners. Heart Lung 2010, 39:304-313.

28. Jastrzebski D, Kozielski J, Zebrowska A: [Pulmonary rehabilitation in patients with idiopathic pulmonary fibrosis with inspiratory muscle training]. [Polish]. Pneumonol Alergol Pol 2008, 76:131-141.

29. Nathan SD, Shlobin OA, Weir N, Ahmad S, Kaldjob JM, Battle E, Sheridan MJ, du Bois RM: Long-term course and prognosis of idiopathic pulmonary fibrosis in the New millennium. Chest 2011, 140:221-229.

30. Richeldi L, Ryerson CJ, Lee JS, Wolters PJ, Koth LL, Ley B, Elicker BM, Jones $\mathrm{KD}$, King TE Jr, Ryu JH, Collard HR: Relative versus absolute change in forced vital capacity in idiopathic pulmonary fibrosis. Thorax 2012, 67:407-411.

31. Anyanwu AC, McGuire A, Rogers CA, Murday AJ: Assessment of quality of life in lung transplantation using a simple generic tool. Thorax 2001 56:218-222.

32. Department of Health: National health service England and wales electronic drug tariff May 2013. ; 2013. 14-6-2013. www.ppa.org.uk/edt/May_2013/ mindex.htm.
33. Joint Formulary Committee: British national formulary (BNF) 65. No. 65. London: British Medical Association and Royal Pharmaceutical Society of Great Britain; 2013

34. Department of Health: NHS reference costs 2011-2012. https://www.gov.uk/ government/publications/nhs-reference-costs-financial-year-2011-to-2012. 811-2012.

35. Mills EJ, Thorlund K, lonnidis JPA: Demystifying trial networks and network meta-analysis. BMJ 2013, 346:f2914.

doi:10.1186/2050-6511-15-63

Cite this article as: Loveman et al:: The effectiveness and costeffectiveness of treatments for idiopathic pulmonary fibrosis: systematic review, network meta-analysis and health economic evaluation. BMC Pharmacology and Toxicology 2014 15:63.

\section{Submit your next manuscript to BioMed Central and take full advantage of:}

- Convenient online submission

- Thorough peer review

- No space constraints or color figure charges

- Immediate publication on acceptance

- Inclusion in PubMed, CAS, Scopus and Google Scholar

- Research which is freely available for redistribution

Submit your manuscript at www.biomedcentral.com/submit
C BioMed Central 